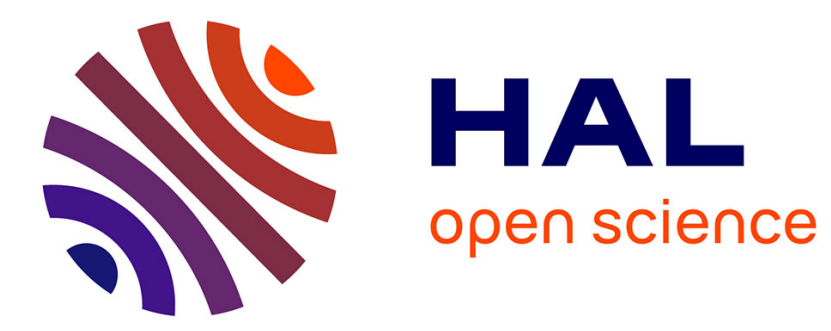

\title{
L'opérateur en en linguistique instructionnelle enactive
}

Didier Bottineau

\section{To cite this version:}

Didier Bottineau. L'opérateur en en linguistique instructionnelle enactive. Langue française, 2013, 2013/2 (178), pp.41-58. halshs-00922762

\section{HAL Id: halshs-00922762 \\ https://shs.hal.science/halshs-00922762}

Submitted on 30 Dec 2013

HAL is a multi-disciplinary open access archive for the deposit and dissemination of scientific research documents, whether they are published or not. The documents may come from teaching and research institutions in France or abroad, or from public or private research centers.
L'archive ouverte pluridisciplinaire HAL, est destinée au dépôt et à la diffusion de documents scientifiques de niveau recherche, publiés ou non, émanant des établissements d'enseignement et de recherche français ou étrangers, des laboratoires publics ou privés. 
Didier Bottineau

CNRS, MoDyCo, Université Paris Ouest Nanterre La Défense

\title{
L'opérateur en en linguistique instructionnelle enactive
}

\begin{abstract}
Résumé
La question de savoir si la préposition en ou les divers types de pronoms en sont munis d'invariants dans leurs catégories grammaticales propres a été âprement débattue. La présente étude s'inscrit dans le cadre des grammaires intructionnelles, qui voient dans les signifiants les activateurs de processus interprétatifs dynamiques plutôt que les marques symboliques qui encodent des représentations mentales, et elle réfère plus précisément à la grammaire enactive. On montre qu'il est possible de proposer pour en un invariant transcatégoriel de nature non-représentationnelle susceptible de s'appliquer à l'ensemble des catégories grammaticales auxquelles ce marqueur s'applique (préposition, préverbe gérondival, clitiques de divers types, et particules parfois mal identifiées).
\end{abstract}

\section{Mots-clés}

En, linguistiques instructionnelles, grammaire enactive, cognition distribuée, préposition, particule, pronom

\begin{abstract}
The question whether the marker en in the various categories in which it appears (as a preposition and as various types of pronouns) has been widely debated. The present study is conducted in the theoretical framework of instructional grammars, which define signifiers as the activators of interpretive processes rather than as symbolic markers encoding mental representations, and it refers more specifically to enactive grammar. It is shown that it is possible to envisage a transcategorial core value of dynamic, non-representational nature, which applies to the whole range of grammatical categories in which this marker is manifested (preposition, gerundive particle, various types of pronouns and "fuzzy" particles).
\end{abstract}

\section{Key-words}

$E n$, instructional linguistics, enactive grammar, distributed cognition, preposition, particle, pronoun

\section{Linguistique instructionnelle et grammaire enactive}

La présente étude se fixe pour objectif de proposer une analyse de en dans la grammaire enactive, laquelle relève du paradigme des linguistiques instructionnelles. Cet ensemble concerne les approches du signifiant linguistique sous toutes ses formes et niveaux de segmentation (submorphème, morphème, unité lexicale, lexies et figements, syntagmes, propositions, textes et discours, proverbes...) qui voient dans les formes langagières non pas l'encodage de représentations préalables (linguistique cognitive), généralement spatiales (Vandeloise 1986, Katz 2002, Sarda 2010) ni la capture des résultats de processus cognitifs sous-jacents (psychomécanique du langage), mais au contraire des «outils», relais ou médiateurs qui permettent d'activer des processus interprétatifs participant à l'élaboration générale du sens. Les approches instructionnelles relient diverses théories par un air de 
famille motivé par cette conception partagée, mais elle émergent au sein de modèles très différents, comme la sémantique computationnelle et représentationnaliste au sein du paradigme cognitiviste (Gosselin 1996 pour la temporalité), le traitement de la forme schématique en syntaxe «temporalisée » dans ou «au voisinage » du cadre de la théorie des opérations énonciatives (la grammaire instructionnelle de Col et al. 2010), la sémantique des formes (Cadiot 1997ab, 2002), la grammaire enactive (Bottineau 2010, 2011, 2012). Pour cette dernière, la parole est définie comme un système comportemental régulé permettant d'activer, pour soi-même (parole réflexive, endophasie) comme pour autrui (parole adressée, exophasie), des chaînes de signifiants correspondant à des processus cognitifs contribuant à la synthèse d'un effet de sens. La grammaire enactive définit la parole comme un protocole interactionnel permettant d'autodéterminer et de structurer des actes de pensée en temps réel en recourant à un protocole d'élaboration ancré dans les rapports intersubjectifs dans leur dimension incarnée. Les lignes qui suivent appliquent cette conception à un élément ciblé, le marqueur en, et elles posent la question, non pas de la représentation ou de la classe de situation à laquelle le marqueur renvoie, mais de l'effet que produit sa survenance à un moment donné en syntaxe de la production et comment cet effet contribue à l'avènement global du sens propositionnel dans le cadre des rapports aux marqueurs environnants et des renégociations interprétatives qui s'ensuivent (section 2), ce qui revient à extrapoler l'approche fonctionnelle de Gougenheim (1950) à tous les emplois de en et avec une valeur intrinsèque différente. Pour suivre, l'étude décline cet invariant aux catégories de la préposition (section 3), du préverbe gérondival (section 4) et des pronoms adverbiaux et clitiques (section 5). L'invariant recherché ici n'est pas un signifié de puissance de type psychomécanique (Guimier 1978, Cervoni 1991), ni une forme schématique (Franckel et Lebaud 1991), mais un schème processuel comparable à celui de Kleiber 1997 pour le gérondif. L'idée même de rechercher un invariant fait depuis longtemps l'objet de controverses parfois ironiques (Gaatone 1980, Peeters 1991). Elle est aux antipodes d'approches qui concluent à l'homonymie de plusieurs en au sein même d'une catégorie comme celle du pronom quantitatif (Hulk 1983). L'approche ici défendue diffère des antérieures par son côté procédural plutôt que représentationnaliste, ce qui la rapproche de celle de Cadiot même si ce dernier récuse explicitement l'idée d'un invariant.

\section{La proposition instructionnelle appliquée à en}

Pour déterminer la contribution opératoire du marqueur en dans la dynamique de l'interprétation, on dispose de deux indices :

(a) - les affinités électives analogiques liant conjointement les signifiants et les signifiés :

Dans le domaine des relations spatiales, le signifiant en se trouve être phonologiquement une partie du signifiant dans. Du côté des signifiés, en et dans sont précisément les deux principaux marqueurs des relations d'intériorité et d'intériorisation abstraite et concrète (vivre en Cévennes / dans les Cévennes). Dans le domaine de l'aspect, le signifiant en partage avec son homophone suffixal verbal -ant une valeur commune d'accomplissement, qui peut être cumulée (le gérondif) ou alterner avec des nuances spécifiques (en $+\mathrm{N} / \mathrm{V}$-ant : le travail en cours / le travail courant). Ce rapprochement morphosémantique se retrouve dans d'autres langues, en particulier avec in et -ing en anglais (in an attempt to / attempting to), en dépit des origines respectives hétérogènes de ces marqueurs (in est prépositionnel alors que -ing résulte de l'amalgame probable d'un suffixe déverbatif -ynge, correspond à l'allemand actuel -ung 《-age » de Heizung «chauffage» et d'une flexion nasale-dentale -nd de participe présent analogue à celle des langues romanes, cf. lebend «vivant»). En basque, pour tout verbe, par exemple ikusi «voir», le participe présent s'obtient en ajoutant au nom déverbal 
ikuste «acte/fait de voir » un marqueur $-n$ reconnu par les diachroniciens comme étant un inessif (locatif) indéterminé archaïque, de même forme que l'inessif actuel applicable aux substantifs pour en faire des locatifs (etxe «maison », etxea « la maison », etxean « à / dans la maison »). On dispose ainsi d'un faisceau de plusieurs langues qui présentent le même isomorphisme partiel ou total entre des marqueurs nominaux de relation spatiale liés à l'intériorité d'un côté, et des marqueurs verbaux de point de vue aspectuel lié au déroulement de l'autre.

Si l'on poursuit à titre heuristique la recherche de telles correspondances, on doit se demander si l'on est fondé à rapprocher le clitique en (en synchronie) de la préposition déjà comparée à -ant. Or il se trouve qu'en frioulan, le clitique partitif correspondant à en, de forme inti, se construit sur le même élément formateur que le gérondif du verbe. L'isomorphisme préposition / clitique observé en français n'est pas non plus un fait isolé et demande à être interrogé comme fait linguistique pertinent. Ces analogies paradigmatiques suggèrent que l'opérateur en réalise un compromis morphosémantique entre la localisation spatiale en intériorité de type dans et le déroulement cursif de type -ant.

(b) - Les valeurs cardinales de en en contexte minimal :

La préposition en entre dans une série de figements utilisables isolément sous prosodie injonctive pour donner l'ordre d'amorcer l'action correspondante, d'entrer en phase exécutoire (En route! En marche! En piste! En avant! En arrière! "Tout le monde en haut! Tout le monde en bas! » En voiture Simone!). Sans une telle prosodie, des figements de même structure sont régulièrement utilisés pour qualifier l'état actuel d'un personnage engagé dans une situation ou en processus: En voyage, En mission. Dans ces exemples choisis pour l'absence d'interférence avec des éléments contextuels explicites, où l'effet de en se laisse échantillonner en réduisant au minimum les nuances parasites issues des autres termes impliqués, en permet de relier un expérienceur humain implicite à un site explicite correspondant à une localisation accessible par un déplacement (haut, bas), un déplacement défini en termes de trajectoire (avant, arrière), de support de trajectoire (route, piste), de mode ou de vecteur de déplacement (marche, voiture). Dans tous ces cas, en crée une relation provisoire entre un processus et un expérienceur affecté (je suis en voyage implique «vous savez en quoi cette activité affecte mon état actuel »; on ne dit pas simplement je voyage, qui porte un point de vue trop extérieur, sauf pour exprimer une généralité) : en réalise une focalisation interne par empathie, oriente l'attention interprétative vers une compréhension de l'affect du sujet (une valeur «qualifiante » pour Amiot \& De Mulder 2001); (être) en vacances $=$ vivre la situation momentanée et être sollicité par ses implications ; avec l'injonction : En route! = «engagez-vous dans le processus dont vous savez comment il vous mobilisera tant qu'il perdurera ». Dans ces exemples restreints, en évoque obligatoirement un processus en déroulement, l'accomplissement d'une mutation, l'inscription dans une phase transitionnelle en principe délimitée ; cet effet est en tout point conforme à la manière dont ant échantillonne un processus en s'appliquant à un radical verbal, et il faut signaler qu'en espagnol la même valeur est obtenue en utilisant le gérondif, qui met en conflit la situation constatée (on ne bouge pas) et la situation évoquée : ¡Andando! 'marchant' «En route!» ou «En avant ! ». Et par sa position introductrice, en dans ces expressions active une relation entre un terme-source implicite ou explicite $\mathrm{X}$ et un terme-cible explicite $\mathrm{Y}$ (comme le fait l'article entre un référent implicite et une notion lexicale explicitée par un signifiant nominal : un chien). L'effet est une mise en mutation transitionnelle du support-source implicite affecté : les propriétés de $\mathrm{X}$ sont modifiées par celles de $\mathrm{Y}$ dans le cadre de la relation active et temporellement limitée activée par l'opérateur en. Cette caractérisation n'est pas suffisante, elle ne rend pas compte de faits de figement et de thématisation culturelle qui autoriseraient en voiture et même randonnées en cheval / en vélo (dépliants touristiques). 
Sur la base de ces observations, pour le moment restreintes à ces expressions confinées, on avance par extrapolation la proposition instructionnelle générale selon laquelle l'effet interprétatif produit par en est comparable à celui de -ant, à savoir, la présentation à la conscience interprétante (sui-réflexive et/ou adressée) de la «représentation » ou simulation mentale d'un processus de déroulement, de transition, ou de transformation / transmutation.

Cette définition a certes une apparence très générale et abstraite, mais elle permet en contexte de faire émerger les valeurs résultantes d'intégration (Kindt 1998) et de relation contenant / contenu (Franckel 1999) mais elle réserve à en (et -ant) une fonction uniquement processuelle et non représentationnelle. Elle prend une dimension très concrète en insistant sur le fait que si l'aspectualité de en peut se retrouver dans la situation évoquée quand les éléments lexicaux notionnels et savoirs culturels s'y prêtent (être en colère), elle peut se limiter à l'acte mental interprétatif d'évocation indépendamment des propriétés des objets ou de l'absence de procès référentiel (une statue en bois = une statue dont je vous fais découvrir la matière, en jouant le rôle d'introducteur heuristique ; la transition est celle du cheminement interprétatif, pas celle du devenir de l'objet; j'en veux = je veux de la chose que nous nous employons à quantifier et/ou qualifier, vs je le veux = je veux l'acte ou l'objet que nous avons prédéfini). Le processus interprétatif prêté à en, inévitablement schématique quand on l'envisage hors contexte, se concrétise in situ en s'appliquant à des domaines spécifiques très divers mais non moins précis, et les lignes qui suivent s'appliquent à les définir. C'est en cela que l'approche est enactive et non représentationnelle, consacrée à la dimension métalinguistique des processus comme le sont la psychomécanique (Guillaume), la théorie des opérations énonciatives (Culioli) et une partie de la grammaire métaopérationnelle, et distincte de la sémantique générale (Pottier) et de la linguistique cognitive grammaticale (Langacker) et sémantique (Talmy) ; et elle fait reposer la dynamique cognitive des processus signifiés par la dynamique même des signifiants. L'intérêt de l'apport enactif est, selon les cas, (i) au minimum, d'améliorer la compréhension de l'origine de certains effets comme l'empathie, déjà identifiés par les théories de l'énonciation en les ancrant dans la dynamique du signifiant, (ii) de dégager des facettes sémantiques innovantes, en matière d'interlocution notamment, (iii) de relier plus clairement les applications figuratives et non figuratives du même invariant processuel. Cette approche suppose que l'on accepte de traiter /ãl grammatical comme un signifiant ou activateur interprétatif muni d'un profil invariant (en amont de toute interaction avec d'autres activateurs) mais démuni de toute catégorisation préalable (préposition, préverbe, clitique, affixe dans des compositions parasynthétiques du type embarquer, enfoiré), les catégories étant problématiques en elles-mêmes (Gaatone 2001 pour la préposition). Les morphèmes transcatégoriels sont communs dans certains types linguistiques (Robert 2003) mais marginaux et atypiques dans les langues à lexique de forme base + flexion comme le français, et en requiert des analyses que l'on ne retrouverait pour aucune autre préposition.

\section{En prépositionnel}

En tant qu'opérateur, en oriente l'interprétant vers l'exécution mentale d'un processus ou de déroulement ou de transition, qui concerne la prise de conscience des propriétés ou de l'état de l'objet $\mathrm{X}$ en fonction du prédicat Y qui lui est reversé, lui-même de nature processuelle ou non : en met en scène la durée de l'accès à une connaissance nouvelle et dirige la formation d'un savoir. Employé dans une fonction prépositionnelle, en position de relateur, l'opérateur en applique ce processus schématique de «transition » à la relation X-Y qu'il instancie dans l'énoncé par sa position syntaxique. 


\subsection{En et les espaces géographiques concrets et abstraits}

Même si cette préposition est régulièrement impliquée dans des énoncés figurant un repérage dans l'intériorité d'un espace confiné plus ou moins abstrait, elle alterne rarement avec dans suivi d'un déterminant : Péril en la demeure (film français de Michel Deville, 1985) vs Péril dans la demeure : $\mathrm{X}$ est en péril si X est dans la demeure, avec défigement de la locution en la demeure, analogie implicite des constructions en péril / en la demeure et assimilation du site demeure au jugement péril implicitement reversé à l'expérienceur humain non mentionné. Le lecteur du titre est amené, en tant que spectateur, à se figurer empathiquement les ressentis émotionnels du personnage fictionnel dans le contexte évoqué.

Ce contraste se retrouve dans nombre d'expressions de la localisation. Les élèves sont dans la classe est une localisation, alors que Les élèves sont en classe leur attribue un engagement processuel typique (on comprend en temps réel qu'ils jouent leur rôle d'élèves), d'où Le chien est dans la/*en classe ; en sélectionne des sujets compatibles avec le rôle stéréotypique qui les recrute à l'interprétation. L'emploi de dans fait du locuteur un simple informateur (l'allocutaire apprend une localisation sans plus), alors que celui de en en fait un prescripteur implicite de comportements possibles ou interdits pour l'allocutaire : en modifie les élèves en fonction de classe et implique « occupés », « indisponibles », « inaccessibles », ce que ne fait pas dans. Ce versant psychologique de l'effet de sens de en dans l'interaction située est directement lié à la façon dont en agit en temps réel sur la connaissance interprétative de l'entité $\mathrm{X}$ en fonction de la propriété $\mathrm{Y}$ : l'imposition du schème de transformation aux états mentaux de l'allocutaire permet d'exercer une influence. Le caractère non-spatial de en (vs Katz 2002) est illustré par le fait que si un «locatif » donné admet la commutation en/dans dès lors que l'alternance localisation / affectation est pertinente, la commutation de sujets avant en mène toujours à des blocages radicaux si le transfert de propriétés ne fait pas sens. On peut passer le week-end dans les Cévennes (locatif), mais si on le passe en Cévennes, en crée un effet de «coalescence»: il donne à ressentir la manière dont cette localisation Cévennes, que l'on sait transitoire en contexte (week-end), affecte sur cette durée le sujet humain qui lui est rattaché ; le contenu de l'affect en question est spécifié par les valeurs culturellement attachées aux «Cévennes » et doit affecter un sujet capable de le recevoir $\left({ }^{*} \mathrm{Ce}\right.$ cheval vit en Cévennes). Ce qu'évoque en Cévennes varie avec les couples interlocutifs et les contextes (en particulier dans le domaine publicitaire : randonnée / équitation / parapente en Cévennes, qui évoquent la particularité de l'expérience de l'activité dans le contexte cévenol plutôt que camarguais ou vosgien), en particulier là où l'alternance en / dans se pratique effectivement (Gard, Héraut). D'autres régions comme Alpes ou Pyrénées n'entrent pas habituellement dans la même construction (*passer le week-end en Alpes), mais on la trouve couramment dans le jargon des annonceurs publicitaires avec cette valeur (ski en Alpes / Pyrénées) dès lors qu'il s'agit d'évoquer la particularité du vécu en situation : l'expression émerge dès lors qu'un consensus sur les valeurs évoquées apparait au sein de la communauté d'usagers, large ou étroite. Devant les noms de pays (En France), en souligne les implications culturelles de la localisation de X même si tous les noms de pays n'emploient pas cette préposition pour des raisons liées au genre, au nombre, à la consonne initiale (ce point est trop riche pour être détaillé ici).

De la même manière, on trouve dans les forums des usages «anormaux » de en qui mettent manifestement en exergue les conditions d'expérienciation de la localisation par un $\mathrm{X}$ animé en rôle de support d'affect : sur vs en autoroute [sic / blog] moi sa va faire 3 ans que j'ai mon permis et j'ai tjrs pas conduit en autoroute - résultat : j'ai peur de l'autoroute lol (= je n'ai pas l'expérience de la conduite sur autoroute). Mesdames et Messieurs, notre TGV est à l'arrêt en pleine voie vs Le chien est en arrêt : en arrêt souligne la façon dont arrêt modifie l'expérience vécue par le chien flairant une piste, d'où *notre TGV est en arrêt. Corollairement, cf. être en repos (éprouvé par un humain) vs être au repos (constaté pour un inanimé). Comme en fait 
interpréter le rapport $\mathrm{X} / \mathrm{Y}$ en temps réel et en situation, la plupart de ces expressions sont polysémiques : je suis en arrêt implique un congé maladie ; selon les contextes, je suis en sécheresse signifie (i) en «crise de foi » religieuse (18-19 siècle), (ii) en souffrance d'une sécheresse physiologique à spécifier (oculaire, dermatologique, vaginale...), (iii) en tant qu'agriculteur, dans une période de restriction locale de l'irrigation par arrêté préfectoral, (iv) en tant qu'écrivain, dans une période improductive, (v) en tant qu' amateur d'œuvres littéraires ou musicales, en manque de trouvailles récentes.

Les intégrations de propriétés X/Y réalisées par en concernent les lieux publics dont les conditions de vie sont culturellement déterminées (être en prison, en maison de retraite, en collège, en lycée, en université) : en commande à l'interprétant d'imaginer l'expérience ou l'affect de $\mathrm{X}$ en fonction des propriétés connues de $\mathrm{Y}$, qu'il s'agisse ou non d'un «lieu » ou « espace » à proprement parler (être en carafe / en galère), ce qui suppose un consensus implicite en la matière. Cette construction à effet empathique ne fonctionne que si les interlocuteurs ont accès une vision partagée et stéréotypée de la teneur de l'affect. L'expression figée sera grand public si ces conditions sont un cliché : être en prison. Elle peut être confinée à un jargon perméable hermétique : être en cuisine (pour un serveur ou un cuisinier, être mobilisé par ce que suppose cette localisation dans le cadre de l'exercice de la profession ou de l'activité (Maman est en cuisine - peut être ironique)). Dans des brochures religieuses: Prions en église, Prions ensemble en église, expression qui souligne la particularité du vécu dans ce cadre particulier. La locution en plein $(e)+\mathrm{N}$ focalise le moment d'une période dont les propriétés intrinsèques atteignent un paroxysme, avec pour l'expérienceur des implications culturellement déterminées (en pleine nuit / voie), positives ou négatives; cette figuration est bien un montage scénique propre au français, que l'on ne retrouve pas dans d'autres langues $(*$ in full night). L'invocation de références culturelles se manifeste souvent par l'indétermination et la stéréotypie générique : être en attente (de traitement) vs être dans l'attente (sens littéral), être en souffrance vs être dans la souffrance. Ceci vaut pour les «activités normalisées » de Leeman 1995 (Les soldats marchaient en silence).

\subsection{Structures d'affect}

Les structures d'affect du type être en colère, largement étudiées, sont très représentatives du processus par lequel en modifie $\mathrm{X}$ en l'intégrant à un état $\mathrm{Y}$ transitoire (par le sens de l'unité lexicale) et fait découvrir et ressentir la modification de $X$ par l'interprétant, créant un effet de partage, d'empathie, de focalisation interne. Alors, pourquoi ne peut-on, comme le montre Danielle Leeman (1995), être en peur? Pour les noms abstraits, l'aspect de l'unité lexicale joue un rôle essentiel (Martinie et Vigier, ce volume), et l'usage révèle des fluctuations importantes. Si être en peur est modérément attesté sur internet (786 occurrences de «je suis en peur » le 5 décembre 2012), pour les hyponymes la fréquence est extrêmement élevée : je suis en stress (289000), je suis en panique (382000), alors que d'autres sont peu attestées (être en trouille, être en trac) et relèvent de l'innovation analogique accidentelle ou expressive dont on ignore si elle est susceptible de gagner du terrain. En fait, il importe de considérer les expressions dans le jeu des réseaux d'oppositions avec ce qu'elles signifient. La construction avoir + adjectif ou nom indéterminé concerne des ressentis physiologiques normaux de la vie courante pour lesquels il est inutile de focaliser un ressenti empathique pour accéder à une compréhension de l'autre : avoir faim, soif, froid, peur. La même construction avec le défini concerne les ressentis ordinaires de la vie interactionnelle socialisée : avoir la trouille, le trac, les chocottes, la honte, le blues. Ceci concerne les maladies (avoir la grippe, les oreillons, le cancer), dont l'expression ne vise pas à communiquer un ressenti empathique de la condition $\mathrm{du}$ malade (*être en grippe, vs prendre quelqu'un en grippe), mais à le repositionner socialement par rapport aux non-malades en matière de conditions de vie. Celle avec en 
amalgame l'état momentané du sujet aux propriétés du prédicat et en livre une reconnaissance empathique : être en colère, pétard, foufelle (régionalisme : Lille et Arras). Un même terme peut se rencontrer dans deux structures : avoir froid (ressenti ordinaire) vs être en froid avec quelqu'un (ressenti spécial que la lexie fait simuler) ; avoir chaud vs être en chaleur (avec des implicites culturels sans lesquels l'expression est inintelligible); avoir faim vs (développements récents) être en faim (je suis en faim : je fais connaître la sensation de faim que j'éprouve) et être en faim de (substitution ludique par rapport à être en fin de pour l'expression d'un désir ardent) : je suis en faim de toi / de vie / d'adolescence / de musique / de vacances / de cul / de retour à la maison. Lorsqu'une lexie comme avoir la trouille est hégémonique, elle ne laisse aucune place à des tentatives comme être en trouille, sauf dans des cas très particuliers où un contexte original le motive, comme une quantification (je suis en trouille totale / complète) ou un niveau émotionnel hors du commun : Et je suis en trouille en pensant à la prochaine fois ou nous allons faire l'amour (énoncé sur un forum relatif à l'impuissance masculine ; dans cet exemple très introspectif, le motif de la «trouille » n'est pas le type d'interaction sociale qui motive ordinairement l'emploi de ce terme); (propos d'une femme violée) Lors de cette nuit, j'ai découvert une douleur immense, un mal que même maintenant je peux encore ressentir lorsque je suis en peur, vis à vis d'un homme. En revanche, la construction empathique s'impose lorsque celle en avoir + défini n'existe pas, et elle est très à la mode (être en stress / panique / crise / anxiété / angoisse) et interactive, se rencontrant bien plus en première personne qu'à la troisième, et très rarement à la deuxième. Etre en peur est rare et ne se rencontre que dans des cas extrêmes (et hautement signifiants) comme celui qui précède, mais être en peur panique est devenu très courant, conformément à la vocation de la construction. Je suis en amour peut être un anglicisme maladroit (surtout suivi de avec), mais il forme également une construction, comme dans la chanson Je suis en amour de Diane Tell (en français québécois). Le français parlé présente couramment des circonstances matérielles comme des affects : Pour le dîner au resto j'aimerais dire oui mais je ne suis vraiment pas en fonds en ce moment. Dans les forums, on trouve marginalement des emplois de en + maladie pour focaliser un ressenti (je suis en rhume, pfff!), le ressenti pouvant être métaphorique (je suis en grippe avec mes parents). Dans tous ces emploi, le sujet humain est modifié par ce qu'introduit en dans des conditions telles que toute autre interaction humaine possible s'en trouve profondément affectée, et l'allocutaire est potentiellement le premier intéressé $:$ je suis en stress $=$ ne vient pas m'embêter, il est en colère $=$ pas à prendre avec des pincettes, etc. Quand en fait prendre conscience de cet état de X à l'allocutaire, il l'invite par là même à en tirer les conséquences pour les éventuelles interactions où il pourrait lui-même se trouver engagé.

\subsection{Matière des objets}

L'expression une statue en bois, avec en comme opérateur de temporalisation de la relation statue / bois, amène l'interprétant à prendre conscience de la matière dont est fait l'objet au moment même où l'expression est formulée: une statue en bois; cette statue est en bois (j'arrive à cette conclusion par l'observation et le toucher, ou je fais parvenir l'allocutaire à cet état de conscience en lui faisant imaginer le voir et le toucher par lequel on en passe pour aboutir à cette conclusion); en «transforme » à l'interprétation une statue immatérielle en objet matériel. Cet effet de sens, non référentiel et appliqué au devenir de la représentation, fait écho à un emploi habituel de en comme introducteur des compléments de verbes de mutation (se) transformer en, se mettre / barrer en; monter en haut, descendre en bas, tourner en rond, s'étaler en flagorneries qui pour leur part traitent d'une transformation effective du sujet avec effet de résultativité (Dagnac 2009), mutation dont la teneur est spécifiée par le verbe. En formule un devenir dont le trajet s'applique soit à des éléments référentiels explicités par le contexte, soit au parcours interprétatif implicite telle que 
l'attribution postérieure d'une matière à un objet. Ceci confère à l'expression en bois une dimension heuristique et sensuelle (alliance en or, couverts en argent vs médaille d'or / d'argent), et bloque l'emploi de en dans tous les cas où il n'est pas question d'interpréter littéralement la matière dont on se dit fait: ${ }^{*}$ je ne suis pas en bois (Pocahontas pourrait dire cela à Grand-Mère Feuillage dans le dessin animé), *ça me laisse en marbre. Par contraste, dans une statue de bois, de, préposition ablative présupposant une origine dépassée, fait de la relation statue / bois un passé énonciatif, une préconstruction qui n'est pas réalisée à l'instant de parole: ceci fait de une statue de bois un objet relevant d'une catégorie connue et reconnue, et du prédicat de bois une propriété dite et redite, comme dans Les petits chanteurs à la croix de bois. Une statue de bois a une connotation plus intellectuelle et distante (par opposition à la connotation empirique et engagée de en bois), aussi les deux tours ne se retrouvent-ils pas dans les mêmes genres discursifs et textuels. L'expression médaille d'or / d'argent n'a pas pour effet de faire ressentir le plaisir éprouvé au contact de ces matériaux nobles, mais de hiérarchiser les médailles en fonction d'un classement en championnat sportif, et de faire symboliser par l'objet une classe de rang (pièce d'or / d'argent, vs *bijou d'or / d'argent). Hors de ce contexte, un archéologue peut effectivement présenter ses trouvailles en parlant de médailles / pièces en or / en argent. Comme précédemment, l'emploi de en a pour l'allocutaire des implications pragmatiques matérielles directes alors que celui de $d e$ s'en tient à l'intellectuel et au symbolique sans engagement par l'action : la reversion par en de la propriété sur le sujet opère une mutation de l'idée que l'interprétant s'en fait et modifie ses perspectives d'intervension ultérieure.

On laisse de côté diverses constructions figées et semi-figées (en avance) et énonciatives (où le X modifié est l'attention interprétative de l'allocutaire : en fait, en réalité), que l'on réserve à des études particulières (Bottineau \& Do-Hurinville, à paraître), et on se tourne vers le cas du gérondif.

\section{En gérondival}

L'analyse bimorphématique du gérondif a été largement débattue, et il s'avère difficile de relier une valeur sémantique de en gérondival au sens prépositionnel (De Mulder et Amiot, ce volume), sauf si l'on s'intéresse à l'effet procédural de en dans la position syntaxique qu'il occupe précisément. En syntaxe linéaire, l'observation montre que le préverbe en occupe une place de sujet, alors que -ant occupe une place de flexion de personne : Nous parlons $d u$ problème / En parlant du problème; Nous ne parlons pas du problème / En ne parlant pas du problème; Nous le voyons / En le voyant; Nous ne le voyons pas / En ne le voyant pas; Nous le lui donnons / En le lui donnant; Nous ne lui donnons pas / En ne le lui donnant pas. Dans tous ces exemples, la place syntaxique du sujet est instanciée soit par un clitique (nous), soit par une marque de relation, en, laquelle stipule que le sujet attendu est en devenir, inscrit dans un processus métalinguistique de transformation, en transit entre un état antérieur et un état postérieur. En profère à l'instant de parole une redite actualisée d'un sujet commun à deux verbes et opère une intégration des actes qui s'interprète différemment selon les contextes (concomitance, manière, causalité, signification de l'acte); avec en, le locuteur assume l'acte de création du rapport, alors que les participales font du lien implicite au sujet une évidence tacite imputable au contexte. Dans la citation de Rabelais Les uns parlaient en mourant, les autres mouraient en parlant, en réitère en position de sujet du verbe mourir un sujet Les uns qui a déjà été défini en tant que sujet du premier verbe parler et qui se trouve redéfini en tant que second sujet: en inscrit un opérateur de transition dans une place syntaxique de sujet. L'ensemble forme ce que l'on peut nommer un sujet transitionnel, dont le rang personnel est indéfini et s'indexe variablement sur celui du sujet-source du premier verbe en amont de la 
transition. Parallèlement, le participe porte un marqueur -ant qui active l'image d'un processus en déroulement et crée un effet d'accord entre le processus en (sujet en devenir) et le processus -ant (procès en devenir, celui spécifié par le radical). La corrélation en / -ant permet ainsi d'harmoniser deux processus cognitifs, celui du retraitement d'un sujet récupéré d'une prédication source et soumis à une transformation, et celui d'une prédication verbale dont l'actualisation est indexée sur celle du sujet transitionnel lui-même; cette analyse transcende le clivage mono- / bimorphématique rappelé par Kleiber 1997. La structure est sophistiquée, mais elle présente l'avantage d'opérer une intégration totale des deux procès conduite en temps réel à l'instant de parole : par la parataxe ou la coordination les uns parlaient et mouraient, les autres mouraient et parlaient, on obtient une juxtaposition de deux procès autonomes avec un doute sur la nature de la relation qui les unit. Avec un simple participe, la seconde proposition se présente comme la suite logique aux yeux de l'observateur de la première (pas nécessairement la conséquence) : Les grandes puissances réunies au sein du G8 à Paris ont écarté faute de consensus l'option militaire pour ralentir les forces de Mouammar Kadhafi, se bornant à promettre pour cette semaine une nouvelle résolution à l'ONU sur des sanctions renforcées. Si on remplace le participe par le gérondif en se bornant, l'effet est une intégration des deux procès en un évènement unique, ici volontaire vu la nature des procès, vs j'ai sali ma chemise en mangeant des spaghettis. Le marqueur d'intégration est indispensable lorsque les évènements ne sont pas liés logiquement par une affinité pragmatique : *j'ai sali ma chemise, mangeant des spaghettis; et ce marqueur analogue à -ant crée un effet d'inclusion du procès principal au procès gérondival qui bloque les inversions si les deux procès ne sont pas censés coïncider strictement : *j'ai mangé des spaghettis en salissant ma chemise vs il devisait doctement en fumant sa pipe / il fumait sa pipe en devisant doctement; le détachement du gérondif amplifie l'effet de cadrage (En revenant de Marquette, j'ai rencontré Toinette). L'emploi du gérondif est compatible avec des relations de consécution non simultanée, voire de rapport de condition nécessaire à effet recherché (Halmøy 2003, Kleiber 2007), mais dans ces emplois en crée un effet de corrélation indissociable et focalise les conditions de transition d'un évènement à l'autre pour le sujet (En apprenant la nouvelle de la capture de ses alliés, le roi de France lève le siège d'Arques; En travaillant plus, tu y arriveras), créant l'impression de procès solidaires, par opposition au participe présent, le coverbe attributif de Herslund (2000). Et lorsque cette solidarité ne va pas de soi, tout est ajouté pour forcer l'intégration de procès ressentis comme incompatibles, créant un effet de réamorçage de la vision du déroulement (Mais Eva [Joly] ne fait pas que se bayrouiser tout en se mélanchonisant), effet que l'on retrouve dans la construction en tout + $\mathrm{N}$ (en toute quiétude / impunité : en dépit de l'attente contraire, comme le suggère l'analyse de la stéréotypie par Anscombre 2001 par rapport à Leeman 1991 : je surveille les enfants, tu peux dormir en toute quiétude [au lieu de t'inquiéter]; il a corrompu le milieu local en toute impunité [au lieu d'être sanctionné]). L'effet intégratif permet en presse journalistique d'improviser des ajouts: Tous veulent "rassurer les investisseurs internationaux». En privatisant leurs services publics. Et en offrant ainsi de nouveaux cadeaux aux marchés. En français parlé, l'intégration peut concerner un procès muni d'un sujet indéfini autre que celui de la principale, ce qui prête à jeu de mots : C'est parce que la fortune vient en dormant que celle-ci arrive si lentement (Alphonse Allais). Sans vouloir réaliser nous-même de jeu de mot, tournons-nous à présent vers le clitique en, réputé homophone de la préposition, mais muni selon nous du même invariant procédural, appliqué à son propre domaine: celui de la formation du syntagme nominal.

\section{En clitique}


Dans un tout autre domaine que celui des relations instanciées par une préposition, en s'utilise en français comme un clitique que l'on peut qualifier d'ablatif, au sens où il figure l'origine d'un mouvement le plus souvent explicitable au moyen de la préposition $d e$. On ne retient pas de distinction stricte entre en quantitatif nominal et en adnominal (Ruwet 1972, Milner 1978, Gaatone 1980, Hulk 1983, Kupferman 1991), Lagae 2001 ayant démontré la perméabilité de la limite entre ces catégories, ni de distinction entre en quantitatif et partitif (Lagae 1994, Kupferman 1999). Le mouvement dont il est question varie avec le contexte :

- mouvement spatial (provenance) : je viens de Lille / j'en viens. De est interprété comme spatial parce qu'il intervient entre un verbe de déplacement avec sujet animé humain et un nom de localisation, mais en soi de n'active que l'image d'un seuil origine d'un mouvement, quelle que soit la nature du seuil et du mouvement. Pour la même raison, en est interprété comme un marqueur anaphorique de provenance, mais ce sens contextuel n'est pas sa vocation propre.

- mouvement conceptuel (quantification par extraction) : je veux du gâteau / j'en veux. Le partitif opère une quantification indéterminée, non spécifique (vs la quantification lexicalement spécifiée: un morceau de gâteau, une part de gâteau), et le processus de quantification schématise l'image d'un prélèvement ou d'une extraction, avec le tout «le gâteau » comme origine de l'extraction; la quantification est grammaticalisée comme un acte conceptuel d'extraction d'une partie issue d'un tout analogue à l'image d'un trajecteur provenant d'une source (en linguistique cognitive). Cet isomorphisme est typologiquement ordinaire : en basque, l'ablatif -tik (Bilbotik nator «j' arrive de Bilbao ») et le partitif - $(r) i k$ (ez dut dirurik «je n'ai pas d'argent) sont en partie constitués des mêmes éléments formateurs.

- mouvement mémoriel (reprise d'une entité-source, d'une notion envisagée en amont): recevoir l'ordre de partir $\rightarrow$ en recevoir l'ordre; tout le monde convient de cela $\rightarrow$ tout le monde en convient; ça vaut la peine de se donner du mal $\rightarrow$ ça en vaut la peine. Ces enchaînements génèrent des constructions au sein desquelles l'unité lexicale d'origine est remplaçable par d'autres qui n'admettent pas la construction en $d e$, ce qui fait perdre de vue le fait que en est conceptuellement ablatif : ça en vaut la chandelle (vs *ça vaut la chandelle de $+\mathrm{INF}$ ) ; je me moque de ce problème $\rightarrow$ je m'en moque, d'où la construction s'en + INF, qui fait sens en elle-même au point que les locuteurs peuvent laisser libre cours à leur imagination pour l'invention du groupe verbal qui doit renvoyer à tout geste corporel sexuel, scatologique, ou absurde (je m'en tartine les aisselles...). Dans tous ces emplois, en active la simulation d'un processus émanant d'une source que l'on peut le plus souvent rétablir, ou du moins gloser, au moyens de de: Je n'en dors plus la nuit / je n'en peux plus impliquent approximativement «(à cause) de ce problème» (mais: *je ne dors plus la nuit de ce problème); je m'en vais (d'ici, de votre présence: je me dégage de l'interlocution, je vous prive de ma présence ; vs je pars : je me transporte en un autre lieu, mais sans mettre l'accent sur cette rupture de la compagnie). Le figement peut opacifier la chose quantifiée ou l'origine évoquée de manière diffuse : j'en ai après lui; je m'en remets à toi (Jacques Brel) (on imagine effectivement se remettre à quelqu'un et de quelque chose, qui ne saurait être spécifié ni même glosé - se démettre de ses responsabilités ? mais là n'est pas l'important : en suggère un schéma transitionnel qui fonctionne même si tous ses termes ne sont pas spécifiés); A en croire les services du ministère de la Défense = approximativement «à croire / si l'on peut croire les services du ministère (sur la base) de leurs affirmations »: à directif «résume » schématiquement une orientation, un choix possible, et en ablatif «résume » une origine identifiée implicitement à un dire émanant d'un locuteur immatériel, «les services ». L'idée d'un choix relatif à un dire-origine dont la fiabilité n'est pas confirmée livre bien le sens global, une adhésion conditionnelle, construite de manière compacte et stylisée. 
A ce stade, on peut considérer que tous ces emplois de en clitique dans ces fonctions hétérogènes (objet partitif, adverbe ablatif, et autres particules plus indéfinissables) sont liés par un processus schématique commun, à savoir la poursuite d'un mouvement conceptuel issu d'une source ou d'une origine, qu'il s'agisse d'un mouvement spatio-matériel (j'en viens), extractif (j'en veux), mémoriel (je m'en souviens; loin de là, il s'en faut), interactionnel (je m'en vais), dialogique (à en croire). Cette valeur processuelle est-elle « la même » que celle postulée pour en prépositionnel dans sa relation analogique avec -ant, et est-on fondé à envisager en synchronie un amalgame morphologique de divers opérateurs issus de sources diachroniques distinctes avec des valeurs hétérogènes ? Considérons à présent deux propriétés de en clitique, l'une sémantique, l'autre formelle.

\subsection{En, un « ablatif conservatif»}

Pour les mouvements spatiaux matériels, le français oppose je viens de là (ablatif de + déictique là, avec effet d'anaphore possible en contexte) et j'en viens (anaphorique par position syntaxique). La formule rhématique de là introduit l'origine d'un mouvement, sans plus. La seconde implique que l'état actuel du sujet est momentanément modifié en fonction du mouvement dans lequel il s'inscrit et défini par son origine, par exemple : je ne vais pas retourner au supermarché, j'en viens (*je viens de là) vs Le couteau, il vient de là (il se range dans ce tiroir) - l'origine spatiale du couteau n'affecte en rien ses propriétés immédiates (et ne l'empêche pas d'y «retourner»). Ce que l'on constate, c'est que en «ablatif », bien que relevant d'une autre classe distributionnelle que la préposition, impose au sujet du verbe de mouvement un effet d'amalgame, lui attribue des propriétés momentanées déterminées par ce rapport cinétique à une source : le sujet est muni de propriétés transitoires du fait d'être engagé dans ce mouvement ; l'emploi de en inscrit le sujet du verbe dans une dynamique de déroulement impliquant une origine et une phase de mutation : en disant j'en viens, je définis je comme étant momentanément muni de propriétés déterminées par le rapport mouvement / origine. La formulation je viens de là a l'effet inverse : elle attribue au sujet des propriétés stabilisées correspondant à l'essence de la source, mais pas des propriétés transitoires immédiates; en conséquence, le verbe venir n'est pas interprété dans son sens littéral de « arriver maintenant», aussi est-il utilisable pour un inanimé (le couteau vient du tiroir) ou, pour un animé, peut-il signifier l'origine sans impliquer un déplacement ponctuel effectif, comme dans la chanson Je viens de là de Grand Corps Malade (2008) : J'viens de là où les mecs traînent en bande pour tromper l'ennui (...) Bien qu'anaphorique, j'en viens est prospectif et a des implications pragmatiques précises pour la suite de l'action et du dialogue, alors que je viens de là est rétrospectif, conformément à l'effet de de. Ceci explique des constructions comme J'en suis! («je m'engage dans l'équipe qui vient d'être évoquée »), $J e$ n'en reviens pas! («je reste affecté par ce que je viens d'apprendre »), Alcatraz, on ne s'en échappe pas; on n'en réchappera pas. L'emploi du clitique produit un effet relationnel d'amalgame et de mutation comparable à celui de la préposition.

\subsection{En, un pronom objet transitionnel}

La différence la plus importante entre un clitique objet ordinaire comme le et l'emploi objet de en est que le est, en principe, saturé, alors que en ne l'est pas : Je veux cette fille (Jacques Higelin) $\rightarrow$ Je la veux \# (on ne peut ajouter de spécification quantitative ou qualitative ; Je la veux belle renverrait à je veux une fille, pas je veux la / cette fille). Avec en, la quantification est soit indéfinie (du gâteau? j'en veux), soit inachevée et à compléter (des bonbons ? j'en veux plein) : le «pronom » en instancie un objet non quantifié ou en attente de quantification. De la même manière, le peut pronominaliser un nom avec ses compléments adjectivaux qualitatifs (je veux l'autre bouteille $\rightarrow$ je la veux), alors que en exclut les qualifications, qui demandent à être explicitée postérieurement (je veux une autre bouteille / j'en veux une 
autre) : le «pronom » en instancie un objet non qualifié, en attente de qualification. Ceci a pu faire dire à certaines grammaires que en n'anaphorise que la notion lexicale (Riegel et al. 1994), laquelle peut être spécifiée a posteriori sous une forme détachée (Gross 1967 : J'en ai vu un, de film) : le «pronom » en instancie également un objet non spécifié notionnellement, en attente de lexicalisation. Ces trois emplois ont un élément en commun : l'incomplétude de l'objet en matière de structuration, faute de quantification, qualification ou lexicalisation, lesquels sont différés, mis en attente par en, ce qui fait de en le marqueur d'un objet en devenir métalinguistique, appréhendé par la forme dans un état transitionnel et inachevé. En active la simulation d'un déroulement qui n'a rien à voir avec des données descriptibles de la situation évoquée (sémantique référentielle ou sémantique cognitive représentationnelle schématique) mais qui concerne la progression cognitive de la formation d'une entité sémantique dont l'élaboration est incomplète au moment où la forme en convoque la prise de conscience. Il arrive même que en dans cette fonction ne soit pas anaphorique et ne renvoie à rien dans le contexte avant ni qui puisse être spécifié : Il faudrait en savoir un minimum sur la vie du prévenu sans s'en remettre à ses seules affirmations. Il n'y a ici aucun effet rétrospectif de présupposition ni d'extraction, juste le fait que l'objet n'est pas prêt à être cliticisé au moment où il a besoin de l'être structuralement, d'où sa mise en attente métalinguistique par l'activateur de transition en et la rhématisation de sa seule propriété quantitative lexicalisée, un minimum.

\section{Conclusion}

La grammaire enactive définit en comme l'activateur d'un effet : l'intégration d'une entitésource à une entité-cible et la modification transitionnelle de la première par le rapport à la seconde. Le rapport source / cible est contextuellement variable : pour la préposition, en relie soit des entités sémantiques explicitement formulées en contexte (un être et une propriété), soit un locuteur implicite à un domaine argumentatif (les locutions prépositionnelles en fonction de connecteurs). Le préverbe gérondival applique cette valeur intégrative à un site syntaxique de sujet, produisant un sujet en devenir constructionnel, métalinguistique ; et le clitique objet ou adverbial applique cette même valeur à des sites syntaxiques d'objet et de complément ablatif, produisant des objets et origines en devenir de même nature. La polysémie et polycatégorialité de en tient au fait que ce marqueur traite de manière cursive des processus dont certains sont à proprement parler sémantiques, observables et explicitables par des moyens lexicaux, alors que d'autres concernent la structuration même des rapports syntaxiques dans la continuité linéaire. On réserve à des études particulières d'autres emplois tels que être en train de (Bottineau 2012).

\section{Références}

Achard-Bayle, G. (2005), « Polysémie de en : diversité et continuité des changements », in P. Dendale (éd.), Le mouvement dans la langue et la métalangue, Recherches Linguistiques, 27, Metz, 243-270.

Amiot, D., De Mulder, W. (2001), «L’insoutenable légèreté de la préposition en », Studii di Linguistica 1, 9-27.

Anscombre, J.-C. (2001), «L'analyse de la construction 'En tout N' par D. Leeman: quelques remarques », Travaux de linguistique 42-43, 183-197. 
Anscombre, J.-C. (2005), «Les deux périphrases nominales Un $N$ en train / Un $N$ en cours : essai de caractérisation sémantique », in Les périphrases verbales, H. Bat-Zeev Shyldkrot \& N. Le Querler (éds), Linguisticaa Investigationes, Supplementa, 25, 103-117.

Berthoz, A. (1997), Le sens du mouvement, Odile Jacob.

Bottineau, D. (2010), « Typologie de la déflexivité », Langages, 178, 2, 89-113.

Bottineau, D. (2010), «Language and enaction », J. Stewart, O. Gapenne, E. Di Paolo (eds), Enaction: toward a new paradigm for cognitive science, MIT, 267-306.

Bottineau, D. (2011), «Parole, corporéité, individu et société : l'embodiment entre le représentationnalisme et la cognition incarnée, distribuée, biosémiotique et enactive dans les linguistiques cognitives », Guignard J.-B. (dir), Linguistique cognitive : une exploration critique, Intellectica 56, 2011/2, 187-220.

Bottineau, D. (2012), «La parole comme technique cognitive incarnée et sociale», Linguistique et phénoménologie du langage, La Tribune Internationale des Langues Vivantes, 52-53, Perros-Guirec : Anagrammes, 44-55.

Bottineau, D. (2012), «Les périphrases verbales 'progressives' en anglais, espagnol, français et gallo : aspect, phénoménologie et genèse du sens », C. Bracquenier \& L. Begioni (dir), L'aspect dans les langues naturelles. Approche comparative, Rennes, Presses Universitaires de Rennes, 93-136.

Cadiot, P. (1997a), Les prépositions abstraites en français, Paris : Armand Colin.

Cadiot, P. (1997b), «Les paramètres de la notion de préposition incolore », Faits de langues, $9,127-134$

Cadiot, P. (2002), «Schémas et motifs en sémantique prépositionnelle : vers une description renouvelée des prépositions dites «spatiales » », Travaux de Linguistique, 44, 1, 9-24.

Cervoni, J. (1991), La préposition, Duculot.

Col G., Aptekman J., Girault S. \& Victorri B. (2010), «Compositionnalité gestaltiste et construction du sens par instructions dynamiques », Cognitextes 5, Numéro spécial AFLiCo 3 Grammaires en Construction(s), consulté le 01/04/2011: http://cognitextes.revues.org/372

Dagnac, A. (2009), «Elle a teint ses rideaux en rouge: entre manière et résultativité », Langages, 175, 67-83.

Danjou-Flaux, N. (1980), «A propos de de fait, en fait, en effet et effectivement». Le Français moderne 38, 110-139.

De Mulder, W. (2008), «En et dans : une question de « déplacement »? », in Olivier B., Prévost S., Charolles M., François, J. \& Schnedecker C. (éds), Discours, diachronie, stylistique du français : études en hommage à Bernard Combettes, Bern : Peter Lang, 277-291.

De Negroni-Peyre, D. (1978), «Nominalisation par être en et réflexivation (admiration, opposition, révolte et rage) », Linguisticae Investigationes, II : 127-164.

Do-Hurinville, D T (2008), «Etude sémantique et syntaxique de en plein $N$ », L'Information grammaticale, 116, 3-9.

Forsgren, M. (2009), "Les connecteurs de fait, en fait, en effet, effectivement : observations empiriques effectuées dans des contextes discursifs variés ». Syntaxe \& Sémantique 10, Rhénania : dimensions syntaxiques et sémantiques, Presses Universitaires de Caen, 51-64.

Franckel, J.-J. (1989), « Être en train de », in Étude de quelques marqueurs aspectuels du français, Genève, Droz.

Franckel J.J., Lebaud D. (1991), «Diversité des valeurs et invariance du fonctionnement de en, préposition et préverbe », Langue française, 91, 56-79.

Gaatone, D. (1980), «La syntaxe de en et l'obsession de la solution unitaire », Lingvisticae Investigationes, 4, 181-201. 
Gaatone, D. (2001), «Les prépositions: une classe aux contours flous », Travaux de linguistique 42-43, 23-31.

Gosselin, L., (1996), Sémantique de la temporalité, Louvain-la-neuve, Duculot.

Gougenheim, G. (1950, 1970), «Valeur fonctionnelle et valeur intrinsèque de la préposition « en » en français moderne », in Etudes de grammaire et de vocabulaire français, Paris : Picard, 55-65.

Guimier, C. (1978), «En et dans en français moderne », Revue des langues romanes, 83 (2), 277-306.

Halmøy, O. (2003), Le gérondif en français, Ophrys.

Herslund, M. (2000), « Le participe présent comme co-verbe ». Langue française 127, 86-94.

Hulk, A. (1983), «La syntaxe du pronom en dans la construction quantitative », Revue québécoise de linguistique 13, 1, 167-199.

Katz, E. (2002), «Systématique de la triade spatiale à, en, dans », Travaux de linguistique, 44 (2), 35-49.

Kindt, S. (1998), «En pleurs vs en pleurant : deux analyses irréconciliables ? », Travaux de linguistique 38, 109-118.

Kleiber, G. (2007), «En passant par le gérondif, avec mes (gros) sabots », Cahiers Chronos, 19, 93-125.

Kupferman, L. (1991), «Aspect du groupe nominal et extraction de en », Le Français moderne, 59, 113-147.

Kupferman L. (1999), « Réflexions sur la partition : Les groupes nominaux partitifs et la relativisation », Langue française, 122, 30-51.

Lagae, V. (1994), «En dans son interprétation partitive : Problèmes de délimitation syntaxique », Revue Romane, 29, 3-16.

Lagae, V. (2001), «Le pronom en: des syntagmes adnominaux aux syntagmes quantificateurs », Travaux de linguistique 42-43, 43-57.

Lamiroy, B. (1991), «Coréférence et référence disjointe : les deux pronoms en », Travaux de Linguistique, 22, 41-65.

Laurendeau, P. (1997), «Concomitance de procès, contingence et agglomérat notionnel : agir / être en », Faits de langues, 9, 145-154.

Leeman, D. (1991), «Les compléments adverbiaux de phrase de type en toute $N$ : En toute objectivité, Paul est un crétin », Études de Linguistique française à la mémoire d'Alain Lerond, Linx ( ${ }^{\circ}$ spécial), Centre de Recherches Linguistiques de l'Université Paris XNanterre, 237-260.

Leeman, D. (1995), « Pourquoi peut-on dire Max est en colère mais non *Max est en peur? Hypothèses sur la construction être en », Langue française, 105, 55- 69.

Leeman, D. (1997), « Sur la préposition en », Faits de langues, 9, 135-145.

Leeman, D. (2012), «Contribution à la définition de la périphrase être en train de », in F. Lautel-Ribstein (dir.), Formes sémantiques, langages et interprétations, Hommage à Pierre Cadiot., La Tribune Internationale des Langues Vivantes, Numéro spécial Juillet 2012, Perros-Guirec : Anagrammes, 133-138.

Milner J.-C. (1978), De la syntaxe à l'interprétation, Paris, Seuil.

Peeters, B. (1991), «Compte rendu de Franckel, Étude de quelques marqueurs aspectuels du français ». Revue canadienne de linguistique 36, 304-307.

Pinchon, J. (1972), Les pronoms adverbiaux 'en' et ' $y$ ', Genève, Droz.

Robert, S. (éd.) (2003), Perspectives synchroniques sur la grammaticalisation. Peeters.

Rossari, C. (1992a), «De fait, en fait, en réalité: trois marqueurs aux emplois inclusifs ». Verbum 3, 139-161.

Ruwet, N. (1970), « Note sur la syntaxe du pronom « en » et d'autres sujets apparentés », Langue française 6, 70-83. 
Ruwet, N. (1972), "La syntaxe du pronom en et la transformation de 'montée du sujet' », Théorie syntaxique et syntaxe du français, Paris, Seuil, 48-86.

Sarda L. (2010). «Les adverbiaux prépositionnels en dans : exploration en corpus de la notion de contenance». CORELA - Espace, Préposition, Cognition, Hommage à Claude Vandeloise - numéro thématique.

Spang-Hansen, E. (1963), Les prépositions incolores du français moderne, Copenhague, G.E.C GAD Forlag.

Vandeloise, V. (1986), L'espace en français, Seuil.

Vigier, D. (2005). Les adverbiaux praxéologiques détachés en position initiale et leur portée. Verbum, XXVII, 2005, 3, 293-312.

Vigier, D. (2008), «Contribution à une étude des constructions antéposées du type : En homme intelligent et humain, il partagea tout de suite l'inquiétude de Marcel (J. Verne)», Discours, 2.

Waugh, L. (1976), «Lexical meaning: the prepositions en and dans in French », Lingua, 59, 69-118. 